\title{
MORFO-ANATOMÍA DE PERIGONIO, GINECEO Y FRUTO EN TRICHOCEREUS CANDICANSY T. PSEUDOCANDICANS (CACTACEAE): IMPLICANCIAS TAXONÓMICAS Y REPRODUCTIVAS
}

\author{
MORPHO-ANATOMY OF PERIGONIUM, GYNOECIUM AND FRUIT \\ IN TRICHOCEREUS CANDICANSY T. PSEUDOCANDICANS (CACTACEAE): \\ TAXONOMIC AND REPRODUCTIVE IMPLICATIONS
}

\author{
Gimena Fernández ${ }^{1}\left(\mathbb{D}\right.$, Laura Stiefkens ${ }^{1,2}\left(\mathbb{D}\right.$, María Laura Las Peñas ${ }^{1,2}(\mathbb{D}$, \\ Adriana Perez ${ }^{2}$ iD N Natalia Delbón ${ }^{1,2 *}$ (D)
}

1. Facultad de Ciencias Exactas, Físicas y Naturales, Universidad Nacional de Córdoba, Avenida Vélez Sarsfield 299, c.c. 495, Córdoba (Argentina).

2. Instituto Multidisciplinario de Biología Vegetal (IMBIV, UNCCONICET). Córdoba, Argentina.

*natalia_delbon@unc.edu.ar

Citar este artículo FERNÁNDEZ, G., L. STIEFKENS, M. L. LAS PEÑAS, A. PEREZ y N. DELBÓN. 2020. Morfo-anatomía de perigonio, gineceo y fruto en Trichocereus candicans y $T$. pseudocandicans (Cactaceae): implicancias taxonómicas y reproductivas. Bol. Soc. Argent. Bot. 55: 557-572.

DOI: https://doi. org/10.31055/1851.2372.v55. $\mathrm{n} 4.29068$

Recibido: 19 Jun. 2020

Aceptado: 23 Oct. 2020

Publicado en línea: 13 Nov. 2020

Publicado impreso: 20 Dic. 2020

Editora: Ana María Gonzalez (D)

ISSN versión impresa 0373-580X ISSN versión on-line 1851-2372

\section{SUMmARY}

Background and aims: Sierras de Famatina are an important area of endemism, where two representatives of Trichocereus coexist, whose taxonomic identity is discussed. Trichocereus. pseudocandicans is endemic, with colorful, diurnal flowers; while, T. candicans has a wider distribution, with white and nocturnal flowers. Taking these differences into account, we carried out a morpho-anatomical study of perigonium, gynoecium and fruit, making qualitative and quantitative comparisons. The objective is to generate new information for the genus, provide reliable taxonomic traits, and make inferences about its reproductive biology.

M\&M: We made temporary and permanent histological preparations of flowers and fruits, using classical techniques. In addition, we analyzed 43 morpho-anatomical variables, which were statistically compared.

Results: The entities present similar morpho-anatomical traits but are differentiated by the color of the perigonium and the total size of the flowers. The following characters stand out: the style presents a stilar canal and transmitter tissue, the receptacle and the ovary wall are notoriously different but intimately fused, even after fertilization, circinate ovules, among others. Only $27.9 \%$ of the variables analyzed resulted in statistically significant differences, the flowers of $T$. candicans being slightly larger than those of T. pseudocandicans.

Conclusions: The similarity found between the taxa reaffirms their high degree of kinship, and it is possible that T. pseudocandicans is a subspecies or hybrid of $T$. candicans. On the other hand, the different floral traits could be due to a high phenotypic variability, influenced by the particular characteristics of the Famatina Sierras.

\section{KEY WORDS}

Cactaceae, Famatina, flower, fruit, morpho-anatomy, Trichocereus.

\section{RESUMEN}

Introducción y objetivos: Las Sierras de Famatina son un área importante de endemismo, allí conviven dos representantes de Trichocereus, de los que se discute su identidad taxonómica. T. pseudocandicans es endémica, con flores coloridas y diurnas; mientras que, T. candicans presenta distribución más amplia, con flores blancas y nocturnas. Teniendo en cuenta estas diferencias, llevamos a cabo un estudio morfo-anatómico del perigonio, gineceo y fruto, realizando comparaciones cualitativas y cuantitativas. El objetivo es generar información novedosa para el género, aportar caracteres taxonómicos confiables y realizar inferencias sobre su biología reproductiva.

M\&M: Se realizaron preparados histológicos temporarios y permanentes de flores y frutos, utilizando técnicas clásicas. Se analizaron y compararon estadísticamente 43 variables morfo-anatómicas.

Resultados: Las entidades presentan características morfo-anatómicas similares, pero se diferencian por el color del perigonio y el tamaño de las flores. Se destacan los siguientes caracteres: el estilo presenta canal estilar y tejido transmisor, el receptáculo y la pared del ovario son notoriamente diferentes pero íntimamente fusionados, incluso luego de la fecundación, óvulos circinótropos, entre otros. Solo el $27,9 \%$ de las variables analizadas resultaron con diferencias estadísticamente significativas, siendo las flores de $T$. candicans levemente mayores que las de $T$. pseudocandicans. 


\begin{abstract}
Conclusiones: La similitud encontrada entre los taxones reafirma su alto grado de parentesco, siendo posible que T. pseudocandicans sea una subespecie o híbrido de $T$. candicans. Por otro lado, los rasgos florales diferentes podrían deberse a una alta variabilidad fenotípica, influenciada por las características particulares que presentan las Sierras de Famatina.
\end{abstract}

Palabras clave

Cactaceae, Famatina, flor, fruto, morfo-anatomía, Trichocereus.

\section{INTRODUCCIÓN}

Las especies endémicas están restringidas a un área geográfica específica y son el resultado de una compleja interacción entre diversos procesos históricos, ecológicos y evolutivos (Young et al., 2002). Son potencialmente más sensibles a las perturbaciones ambientales, y por lo tanto, más vulnerables a la extinción (Godoy-Bürki et al., 2014). En Sudamérica, una de las áreas de endemismo más importante es el sur de los Andes Centrales, desde el sudoeste de Bolivia al noroeste de Argentina, allí la mayoría de las especies endémicas se hallan en ambientes semiáridos, entre 1500-3500 msnm (Young et al., 2002; Aagesen et al., 2012; Godoy-Bürki et al., 2014). En esta zona, la familia Cactaceae se encuentra bien representada, siendo la segunda en cantidad de especies (Aagesen et al., 2012). Se considera que el origen de la familia es Sudamericano y que en los Andes Centrales se llevó a cabo una temprana diversificación de las tribus Trichocereeae y Opuntioideae (Nyffeler, 2002; Griffith \& Porter, 2009).

Formando parte de esta extensa cadena montañosa, un área importante de endemismo y punto crítico de diversidad es el Sistema Sierra de Famatina (La Rioja, Argentina), el cual está habitado por 28 especies endémicas de Angiospermas y numerosas nativas, que crecen hasta los $4000 \mathrm{msnm}$ (Aagesen et al., 2012; Barboza et al., 2016). Presenta una gran complejidad en su biota, la cual probablemente tuvo una evolución aislada e independiente debido a su origen extra andino, por este motivo se ha planteado la necesidad de dar alta prioridad de conservación (Barboza et al., 2016; Godoy-Bürki et al., 2014).

En Famatina habitan 28 especies de la familia Cactaceae, siendo siete del género Trichocereus (Berg.) Riccob. (Tribu Trichocereeae - Subtribu Trichocereinae; Barboza et al., 2016). Este género agrupa a una variedad de cactus columnares, con pilosidad en las areolas florales y en general presenta flores blancas y nocturnas; incluye aproximadamente 45 especies, pero su delimitación ha estado históricamente en discusión (Kiesling, 1978; Anderson, 2001; Hunt et al., 2006; Kiesling et al., 2008; Schlumpberger \& Renner, 2012). Análisis filogenéticos con datos morfológicos y de ADN posicionaron a Trichocereus como monofilético, si también se incluyen dos especies del género Harrisia (Albesiano \& Terrazas, 2012). Por otra parte, Schulumpberger \& Renner (2012) profundizaron el estudio en Echinopis mediante una filogenia molecular usando tres loci de cloroplasto (trnS-trnG, trnL-trnF y rpl16) dividiendo a Echinopsis sensu lato, en 10 grupos entre los cuales podemos destacar los clados Echinopsis, Harrisia, Helianthocereus (donde se encuentran incluidos los taxones aquí analizados), Lobivia, y Trichocereus sensu stricto.

Dentro de este género conflictivo, existen dos representantes de los cuales se discute su identidad taxonómica, Trichocereus candicans (Gillies ex Salm-Dyck) Britton \& Rose y T. pseudocandicans Backeb. ex R. Kiesling. Este último taxón constituye un endemismo restringido, ya que habita solamente en la provincia de La Rioja, en las Sierras de Famatina, entre los matorrales bajos con suelo desnudo (Kiesling, 1978; Kiesling et al., 2008). Trichocereus pseudocandicans, junto a $T$. vatteri R. Kiesling, son las únicas especies endémicas de Famatina con flores coloridas y diurnas, y presentan alto riesgo de extinción (Aagesen et al., 2012; Godoy-Bürki et al., 2014; Barboza et al., 2016). En cambio, T. candicans presenta una distribución más amplia, en las provincias de La Rioja, San Juan, Mendoza, Córdoba, San Luis, La Pampa y Buenos Aires; pudiendo habitar desde los cerros bajos de la precordillera y de las Sierras Pampeanas, hasta zonas llanas pedregosas o inclusive salinas (Kiesling, 1978; Kiesling et al., 2008).

Kiesling (1978) considera a estas entidades como especies diferentes, criterio adoptado en el presente trabajo, y ha propuesto que $T$. pseudocandicans podría ser una especie de reciente formación, derivada de $T$. candicans, presumiblemente un híbrido entre $T$. 


\section{G. Fernández et al. - Morfo-anatomía de Trichocereus (Cactaceae)}

candicans y T. vatteri. Hunt et al. (2006) proponen que ambos taxones son sinónimos; en tanto que, Schlumpberger \& Renner (2012) y Albesiano \& Terrazas (2012) en sus filogenias incluyen solamente ejemplares de T. candicans. Recientemente, Albesiano (2015) en su tesis doctoral concluye que las diferencias exomorfológicas entre ellas son suficientes para considerar a T. pseudocandicans como una subespecie de $T$. candicans.

$\mathrm{Si}$ bien las especies son similares, presentan algunas características distintivas, como la forma de las espinas y el número de costillas. Asimismo, la mayor diferenciación se observa en los órganos reproductivos: las flores de $T$. candicans poseen tépalos internos blanco níveo, son de gran tamaño, con $18-23 \mathrm{~cm}$ de longitud y $11-19 \mathrm{~cm}$ de diámetro, presentan forma acampanada con aroma similar a jazmín o rosas y abren sólo una noche; el fruto mantiene el perigonio seco adherido. Las flores en $T$. pseudocandicans poseen tépalos internos de amarillo hasta rojo, presentan menor tamaño, con $15-18 \mathrm{~cm}$ de longitud y $11-14 \mathrm{~cm}$ de diámetro, son infundibuliformes e inodoras y permanecen abiertas durante dos o tres días; el frutos pierde el perigonio al madurar (Kiesling, 1978; Albesiano, 2015).

Haciendo hincapié en estas diferencias, se propone efectuar un estudio morfo-anatómico del perigonio, gineceo y fruto de $T$. candicans y T. pseudocandicans, realizando comparaciones cualitativas y cuantitativas, con el objetivo de generar información novedosa para el género. Además, siendo estos órganos variables dentro de la familia, se pretende aportar caracteres confiables que ayuden a la delimitación taxonómica de las dos entidades y realizar inferencias sobre algunos aspectos de su biología reproductiva.

\section{Materiales y Métodos}

Área de estudio y material vegetal. Famatina (La Rioja, Argentina) es un cordón montañoso incluido en los dominios fitogeográficos Chaqueño y el Andino-Patagónico. Presenta un clima seco y cálido, con gran amplitud térmica, tanto diaria como anual, alta radiación solar, suelos empobrecidos y una vegetación típicamente xerófita (Aceñolaza, 1996; Barboza et al., 2016). En esta región se pueden encontrar coexistiendo los dos taxones analizados en el presente trabajo, Trichocereus candicans (Gillies ex Salm-Dyck) Britton \& Rose y T. pseudocandicans Backeb. ex R. Kiesling. Los estudios se realizaron en nueve ejemplares en total, 3 de $T$. candicans y 6 de T. pseudocandicans, los individuos elegidos fueron adultos saludables que tuvieran como mínimo dos flores y/o frutos jóvenes. Se recolectaron en tres poblaciones diferentes, ubicadas entre los 1700 y $2100 \mathrm{msnm}$. Los respectivos ejemplares se encuentran depositados en el Herbario de Córdoba (CORD), los datos de recolección son los siguientes: T. pseudocandicans, Las Peñas et al. 593, 605, 606 - 27/ XI/2015. T. candicans, Las Peñas et al. 594, 603,604 - 27/ XI/2015; 616 - 28/ XI/2015; 618, 631 $-29 / \mathrm{XI} / 2015$.

Metodología. De cada individuo se recolectaron entre 2 y 4 flores y frutos en diferentes estadios de desarrollo, los cuales fueron fijados en FAA (Formol, Ácido Acético y Alcohol). Bajo lupa se les realizó un análisis exomorfológico exhaustivo, efectuando una descripción detallada de cada porción y órgano. Además, se midió el largo total del estilo, se registró el número de lóbulos estigmáticos de cada uno y se contaron los tricomas de las escamas florales.

Posteriormente, se realizaron cortes transversales a mano alzada de 5 escamas, tépalos externos, intermedios e internos de cada flor. En tanto que se realizaron 5 preparados de zona alta, media y baja del estilo, y del ovario o fruto. Los cortes fueron teñidos con azul astral y fucsina básica y luego montados con glicerina diluida al $50 \%$.

Además, se confeccionaron 5 preparados permanentes de cortes transversales de estigma, zonas del estilo, ovario y fruto joven. Para su realización se utilizaron técnicas histológicas clásicas con pequeñas modificaciones (Kraus et. al., 1998; Zarlavsky, 2014). Las muestras se deshidrataron en una serie de alcohol etílico en graduación creciente (de $50^{\circ}$ a $100^{\circ}$ ) durante una hora cada paso. Posteriormente se clarificaron con dos pasos de xilol puro de una hora cada uno y se incluyeron en Paramat de la siguiente forma: 3:1 de xilol-Paramat (5 h), 3:1 Paramat-xilol (18 h), Paramat (24 h), Paramat (24 h). A los ovarios y frutos jóvenes se los sometió además a una serie de inclusiones en Paramat por una semana. Con el material incluido se realizaron cortes transversales seriados de 12 a 15 micras de espesor con micrótomo de rotación tipo Minhot, se tiñeron con azul astral y fucsina básica y se montaron con bálsamo de Canadá artificial. 
En los preparados temporarios y permanentes realizados se llevaron a cabo conteos y mediciones utilizando un ocular micrométrico, realizando 5 repeticiones de cada sección de la flor y fruto. Los rasgos analizados fueron:

Escamas y tépalos externos: se tomaron medidas del ancho y espesor de cada uno, se midió el espesor de la epidermis e hipodermis de las caras adaxial y abaxial, se registró el número de conductos secretores y de hacecillos.

Estilo y estigma: el estilo se dividió en tres zonas, alta (inmediatamente debajo del estigma), media (porción intermedia) y baja (sobre el ovario) y en cada una se midió el diámetro total, espesor de la pared estilar, espesor de epidermis e hipodermis, y se contabilizó el número de conductos y de haces vasculares.

Receptáculo, ovario y fruto: se registró el espesor de la epidermis e hipodermis y el espesor de las distintas zonas, receptáculo, pared del ovario, clamidocarpo y pericarpo.

Los datos obtenidos fueron procesados con el programa Infostat (Di Rienzo et al., 2017), se calcularon las medias y los desvíos estándares y se realizaron análisis de varianza (ANOVA) usando un diseño con estructura anidada, para comprobar si existen diferencias significativas entre las especies. Previamente se verificaron los supuestos de homogeneidad de medias y normalidad, en los casos en que algún supuesto no se cumplió, se transformaron los datos con $\log 10$ o se realizó un análisis de la varianza no paramétrico mediante la prueba de KruskalWallis.

Las fotomicrografías de los cortes se tomaron con microscopio Olympus BX61 software Cytovision ${ }^{\circledR}$ (Leica Biosystems); y con lupa Olympus SZX16 con una cámara Olympus DP71.

\section{Resultados}

\section{Caracteres morfológicos}

Trichocereus candicans y T. pseudocandicans presentan una exomorfología similar (Fig. 1A, D). Las flores son vistosas y de grandes dimensiones, actinomorfas, acampanadas y con muchas piezas florales (Fig. 1C, F).

Poseen numerosas escamas dispuestas en la cara externa del ovario y en la zona baja del tubo floral, de color verdoso-amarillento, forma subulada y gran espesor, siendo gruesas y carnosas (Fig. 1B, E). Cada escama lleva en su axila grupos de tricomas eglandulares, pluricelulares, uniseriados, de color castaño claro, abundantes en ambas especies (Tabla 1). Los tépalos son numerosos, fusionados en la base de la flor formando un tubo floral (Fig. 1B, E), y libres en el ápice; se distinguen tres tipos, ubicados cada uno en un ciclo: externos, intermedios e internos, diferenciados por su tamaño, espesor y color (Fig. 1C, F). Los externos son sepaloides anaranjadorojizos, de forma linear-lanceolados con base ancha. Los internos son petaloides espatulados, más largos y anchos, y menos gruesos. Los intermedios poseen características que varían entre los externos e internos. Los tépalos internos e intermedios presentan diferentes colores según la especies, siendo siempre blanco níveo en $T$. candicans (Fig. 1C) y desde amarillo a rojo en $T$. pseudocandicans (Fig. 1F).

Las flores son monoclinas o hermafroditas, de ovario ínfero (Fig. 1B, E). El gineceo presenta un estilo de grandes dimensiones, con una longitud total aproximada de $11 \mathrm{~cm}$ (Tabla 1), sobrepasando la altura de los estambres y tépalos (Fig. 1C, F). El estigma está formado por numerosos lóbulos estigmáticos, siendo en promedio 18 para $T$. candicans y 20 para $T$. pseudocandicans (Tabla 1). Poseen abundantes estambres (alrededor de 200), dispuestos en dos ciclos con diferentes alturas. Los estambres superiores son largos, llegando casi hasta la altura del estilo, dispuestos en una sola fila. Los estambres inferiores son más numerosos y dispuestos en espiral, son cortos y llegan solamente hasta la mitad del tubo floral. Los filamentos de ambos tipos de estambres se encuentran fusionados a la pared del tubo floral. Las anteras son bitecas y basifijas.

Los frutos son globosos, carnosos, con dehiscencia lateral longitudinal. Externamente mantienen las escamas, aunque son más pequeñas que en la flor; son color verde con tonos rosas a amarillo y con tricomas en sus axilas (Fig. 1GI). En su interior presentan numerosos funículos blancos y gran cantidad de semillas negras, de pequeño tamaño. Los frutos de $T$. candicans poseen el perigonio seco adherido (Fig. 1H), mientras que, los de T. pesudocandicans no (Fig. 1I). 


\section{G. Fernández et al. - Morfo-anatomía de Trichocereus (Cactaceae)}
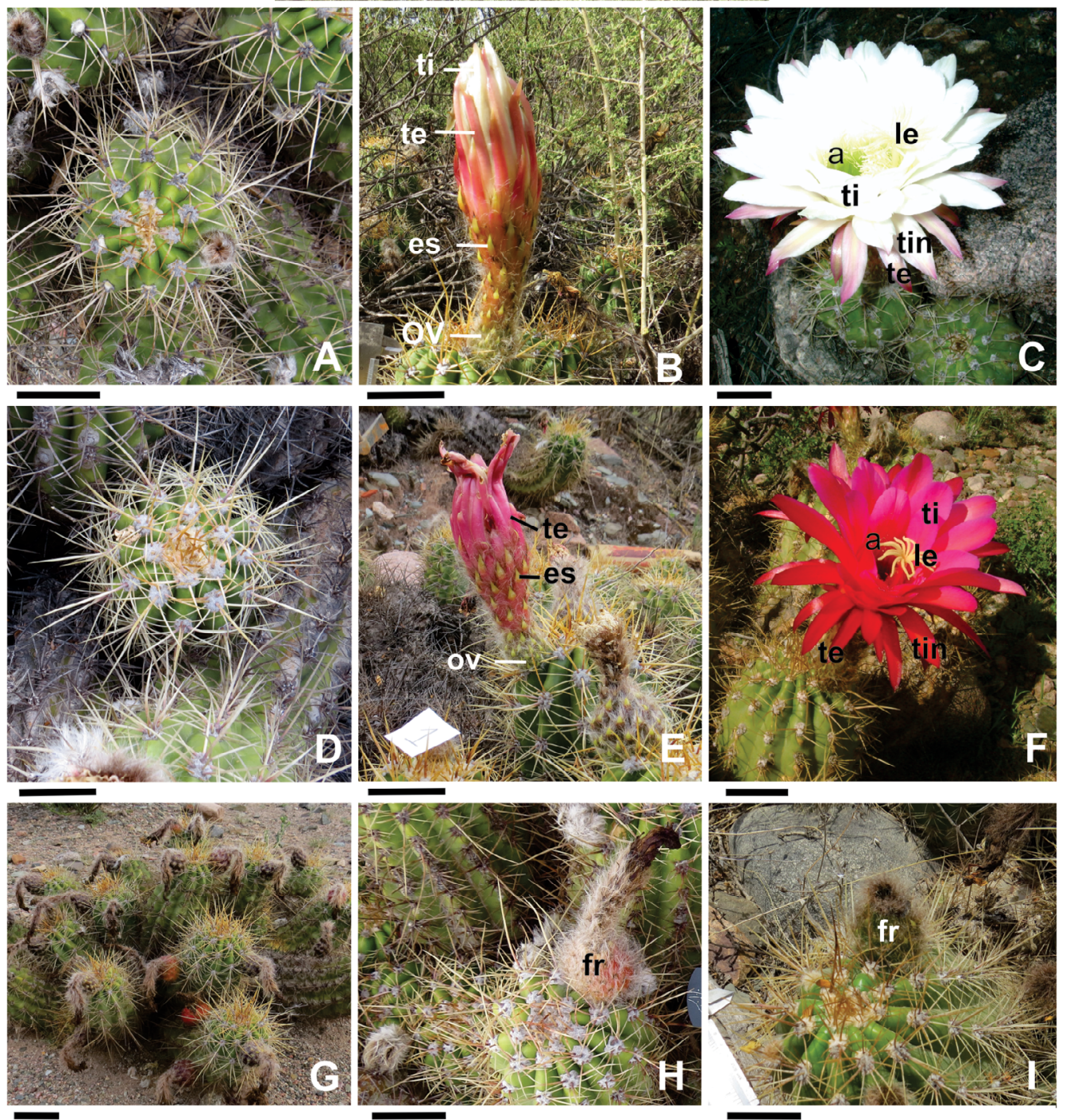

Fig. 1. Hábito, flor y fruto de especies de Trichocereus. A-C, G-H: T. candicans. D-F, I: T. pseudocandicans. A, D: Vista general de la planta. B, E: Botón floral. C, F: Flor abierta. G: Planta con numerosos frutos jóvenes. H-I: Fruto joven. Abreviaturas= a: anteras, es: escama, fr: fruto joven, le: lóbulo estigmático, ti: tépalo interno, tin: tépalo intermedio, te: tépalo externo, ov: ovario. Fotografías tomadas por Las Peñas, M. L. Escalas $=5 \mathrm{~cm}$.

\section{Caracteres anatómicos}

Las flores y los frutos de ambas especies estudiadas son similares, pero con particularidades en cada órgano analizado, los que se describen a continuación.

Escamas: poseen una estructura anatómica similar en las especies estudiadas (Fig. 2A-D). La epidermis es uniestratificada, colenquimatosa, con cutícula algo engrosada y escasos estomas en la cara abaxial (Fig. 2B, D). Inmediatamente por debajo se ubica una hipodermis uniestratificada de células más grandes que las epidérmicas, pero con paredes delgadas (Fig. 2B, D). El mesófilo es homogéneo y compacto, las células de parénquima esponjoso son grandes e isodiamétricas (Fig. 2A-D). Los hacecillos se ubican hacia la cara adaxial, son pequeños, del tipo colateral cerrado y rodeados de una vaina de 
Bol. Soc. Argent. Bot. 55 (4) 2020

Tabla 1. Características cuantitativas en Trichocereus candicans y T. pseudocandicans. Las variables de cada órgano se indican como promedios \pm desvío estándar. * indican diferencia significativa entre las especies, para un Análisis de la Varianza con $p \leq 0,05$

\begin{tabular}{|c|c|c|c|}
\hline \multicolumn{2}{|c|}{ Variable } & \multirow{2}{*}{$\frac{\text { T. candicans }}{115,47 \pm 43,56}$} & \multirow{2}{*}{$\frac{\text { T. pseudocandicans }}{98,47 \pm 33,27}$} \\
\hline \multirow{9}{*}{ Escama floral } & $\mathrm{n}^{\circ}$ de tricomas & & \\
\hline & ancho $(\mathrm{mm})$ & $3,66 \pm 1,19$ & $3,84 \pm 1,14$ \\
\hline & espesor (mm) & $0,97 \pm 0,20$ & $0,9 \pm 0,18$ \\
\hline & epidermis adaxial $(\mu \mathrm{m})$ & $32,87 \pm 9,61$ & $29,19 \pm 9,88$ \\
\hline & epidermis abaxial $(\mu \mathrm{m})$ & $34,1 \pm 7,03$ & $30,91 \pm 7,72$ \\
\hline & hipodermis adaxial $(\mu \mathrm{m})$ & $35,94 \pm 17,47$ & $28,21 \pm 8,31$ \\
\hline & hipodermis abaxial $(\mu \mathrm{m})$ & $33,73 \pm 12,11$ & $40,23 \pm 17,12$ \\
\hline & $\mathrm{n}^{\circ}$ de conductos & $6,7 \pm 2,28$ & $8,4 \pm 3,36$ \\
\hline & $\mathrm{n}^{\circ}$ de hacecillos & $10 \pm 3,45$ & $9,73 \pm 2,09$ \\
\hline \multirow{8}{*}{ Tépalo externo } & ancho $(\mathrm{mm})$ & $6,96 \pm 2,22$ & $7,04 \pm 2,09$ \\
\hline & espesor (mm) & $1,21 \pm 0,27$ & $1,17 \pm 0,22$ \\
\hline & epidermis adaxial $(\mu \mathrm{m})$ & $43,55 \pm 13,46$ & $38,52 \pm 4,37$ \\
\hline & epidermis abaxial $(\mu \mathrm{m})$ * & $40,23 \pm 10,53$ & $33,61 \pm 9,82$ \\
\hline & hipodermis adaxial $(\mu \mathrm{m})$ & $42,32 \pm 15,84$ & $39,25 \pm 13,75$ \\
\hline & hipodermis abaxial $(\mu \mathrm{m})$ & $41,09 \pm 15,91$ & $35,57 \pm 11,36$ \\
\hline & $\mathrm{n}^{\circ}$ de conductos & $10,13 \pm 2,54$ & $11 \pm 3,61$ \\
\hline & $\mathrm{n}^{\circ}$ de hacecillos & $23,77 \pm 7,39$ & $26,27 \pm 7,64$ \\
\hline \multirow{3}{*}{ Estilo } & largo $(\mathrm{cm})$ & $10,75 \pm 2,23$ & $11 \pm 2,96$ \\
\hline & $\mathrm{n}^{\circ}$ de lóbulos del estigma * & $18,33 \pm 1,40$ & $20 \pm 1,46$ \\
\hline & $\mathrm{n}^{\circ}$ de hacecillos * & $18,67 \pm 1,40$ & $19,67 \pm 0,49$ \\
\hline \multirow{5}{*}{ Estilo alto } & diámetro $(\mathrm{mm})^{*}$ & $3,67 \pm 0,63$ & $3,25 \pm 0,34$ \\
\hline & epidermis $(\mu \mathrm{m})$ * & $49,19 \pm 11,24$ & $39,25 \pm 4,09$ \\
\hline & hipodermis $(\mu \mathrm{m})$ * & $48,33 \pm 12,37$ & $41,46 \pm 6,29$ \\
\hline & pared estilar $(\mathrm{mm})$ * & $0,81 \pm 0,09$ & $0,75 \pm 0,06$ \\
\hline & $\mathrm{n}^{\circ}$ de conductos * & $48,83 \pm 21,02$ & $26,87 \pm 11,22$ \\
\hline \multirow{5}{*}{ Estilo medio } & diámetro (mm) & $3,34 \pm 0,68$ & $3,01 \pm 0,56$ \\
\hline & epidermis $(\mu \mathrm{m})$ & $47,84 \pm 10,67$ & $46,61 \pm 8,19$ \\
\hline & hipodermis $(\mu \mathrm{m})$ & $50,54 \pm 15,19$ & $47,59 \pm 13,87$ \\
\hline & pared estilar $(\mathrm{mm})$ * & $0,92 \pm 0,20$ & $0,85 \pm 0,19$ \\
\hline & $\mathrm{n}^{\circ}$ de conductos * & $38,33 \pm 15,20$ & $25,4 \pm 10,34$ \\
\hline \multirow{5}{*}{ Estilo bajo } & diámetro (mm) & $3,61 \pm 0,68$ & $3,24 \pm 0,57$ \\
\hline & epidermis $(\mu \mathrm{m})$ & $40,6 \pm 11,78$ & $35,08 \pm 7,73$ \\
\hline & hipodermis $(\mu \mathrm{m})$ & $38,15 \pm 11,97$ & $37,54 \pm 7,89$ \\
\hline & pared estilar (mm) & $1,11 \pm 0,31$ & $1,01 \pm 0,29$ \\
\hline & $\mathrm{n}^{\circ}$ de conductos & $29,67 \pm 14,67$ & $38,87 \pm 17,05$ \\
\hline \multirow{3}{*}{ Receptáculo } & epidermis $(\mu \mathrm{m})$ & $37,78 \pm 12,49$ & $45,63 \pm 16,85$ \\
\hline & hipodermis $(\mu \mathrm{m})$ & $52,75 \pm 21,22$ & $57,65 \pm 29,98$ \\
\hline & pared del receptáculo (mm) & $3,71 \pm 0,44$ & $3,51 \pm 0,31$ \\
\hline Ovario & pared del ovario $(\mathrm{mm})$ & $2,61 \pm 0,80$ & $2,57 \pm 1,43$ \\
\hline \multirow{4}{*}{ Fruto } & epidermis $(\mu \mathrm{m})$ & $40,97 \pm 11,18$ & $46,12 \pm 12,12$ \\
\hline & hipodermis $(\mu \mathrm{m}) *$ & $64,77 \pm 27,82$ & $83,41 \pm 18,38$ \\
\hline & clamidocarpo (mm) & $4,7 \pm 1,22$ & $4,7 \pm 0,57$ \\
\hline & pericarpo $(\mathrm{mm}){ }^{*}$ & $2,71 \pm 1,04$ & $3,69 \pm 1,45$ \\
\hline
\end{tabular}




\section{G. Fernández et al. - Morfo-anatomía de Trichocereus (Cactaceae)}

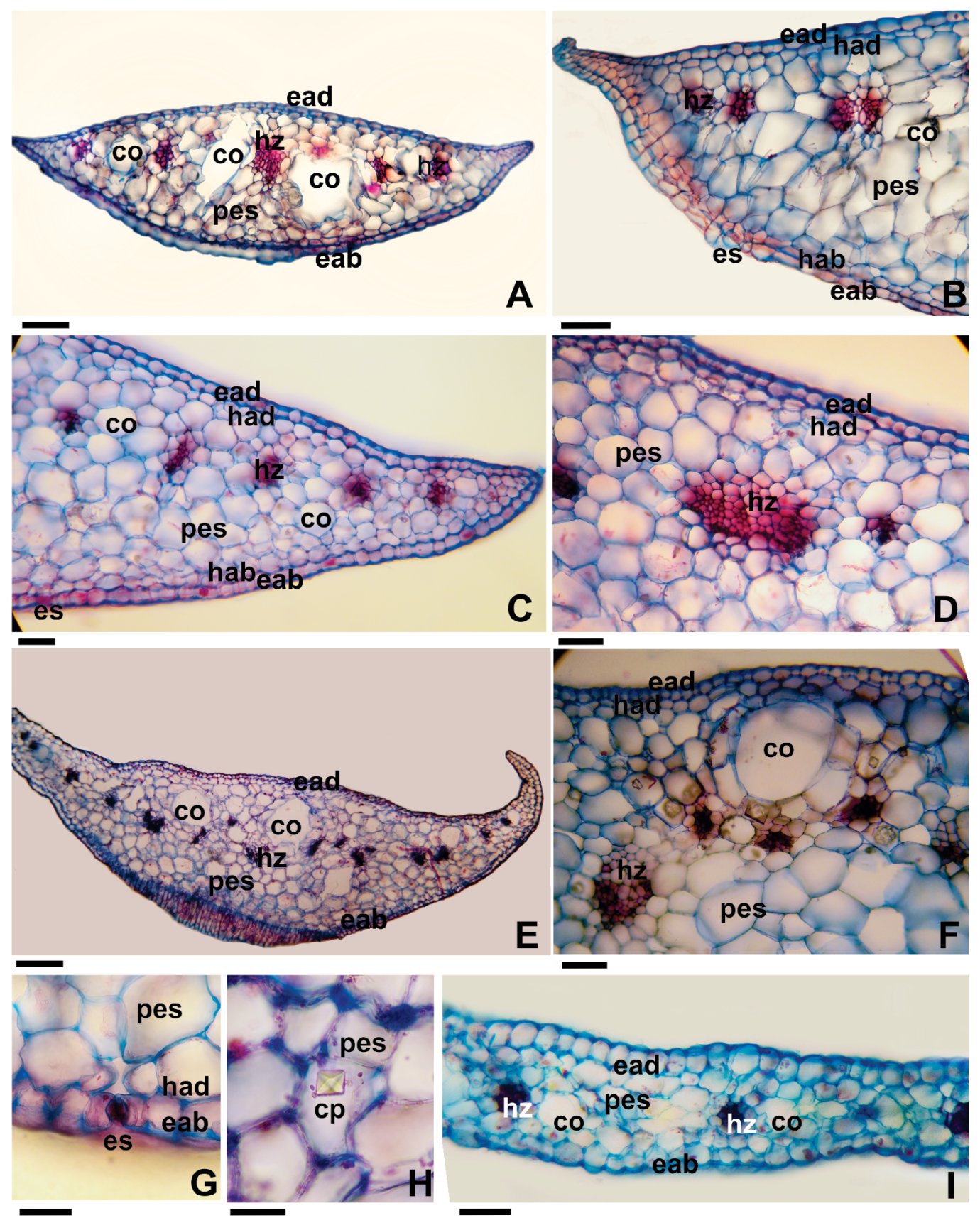

Fig. 2. Escamas y tépalos de Trichocereus en corte transversal. A-B, E-F, I: $T$. candicans. C-D, G-H: $T$. pseudocandicans. A-D: Escama. E-H: Tépalo externo. I: Tépalo interno. A: Escama en vista completa con numerosos haces y conductos, B: Borde de la lámina. C: Porción del extremo de una escama, D: Zona central con hacecillo dorsal medio. E: Tépalo externo en vista completa, F: Detalle de zona con conducto secretor, G: Detalle de un estoma en epidermis abaxial, H: Idioblasto con cristal prismático en célula del parénquima esponjoso. I: Tépalo interno, zona cercana al borde de la lámina con varios haces y conductos. Abreviaturas $=c 0$ : conducto secretor, $\mathrm{cp}$ : cristal piramidal, eab: epidermis abaxial, ead: epidermis adaxial, es: estoma, hab: hipodermis abaxial, had: hipodermis adaxial, hz: haz, pes: parénquima esponjoso. Escalas $=$ A, E: $200 \mu \mathrm{m}$. B-D, F, I: $100 \mu \mathrm{m}$. G-H: $50 \mu \mathrm{m}$. 
células parenquimáticas poco diferenciada (Fig. 2B), el haz central destaca por su mayor tamaño (Fig. 2D). Presentan conductos secretores, idioblastos con cristales conglomerados y piramidales (Fig. 2A, C).

Con respecto a los análisis cuantitativos, ninguna de las variables analizadas mostró diferencias estadísticamente significativas entre las especies (Tabla 1); aunque en la mayoría de las mediciones los resultados fueron mayores en T. candicans.

Tépalos: son similares entre las especies y poseen estructura foliar semejante a la de las escamas (Fig. 2E). La epidermis e hipodermis son uniestratificadas, de células pequeñas en los tépalos externos (Fig. 2F-G) y algo mayores en los tépalos internos (Fig. 2I). La epidermis abaxial presenta escasos estomas (Fig. 2G). Las células del parénquima esponjoso son irregulares en los tépalos externos (Fig. 2E-F); en tanto que en los internos fueron más redondeadas, grandes y distribuidas en pocos estratos (Fig. 2I). Los hacecillos son numerosos y pequeños, ubicados hacia la cara adaxial en los tépalos externos (Fig. 2E) y en la línea central en los internos (Fig. 2I). Son del tipo colateral, acompañado por conductos secretores (Fig. 2F) y algunos idioblastos con cristales (Fig. 2H).

Se encontraron diferencias significativas solamente en una variable analizada, el espesor de la epidermis abaxial resultó mayor en $T$. candicans. Si bien el resto de las variables no fueron estadísticamente diferentes, se observó que la mayoría de ellas presentaron valores mayores en $T$. candicans. Por su parte, todas las mediciones realizadas, en ambas especies, resultaron mayores en los tépalos que en las escamas (Tabla 1).

Estilo y estigma: en corte transversal se observó que cada lóbulo estigmático está rodeado de un tejido epidérmico papiloso. Inmerso en un tejido parenquimático muy laxo se encuentra un solo haz vascular de escasas dimensiones, rodeado por algunos conductos secretores (Fig. 3A). El estilo presenta forma circular (Fig. $3 \mathrm{D}, \mathrm{G})$, con un diámetro aproximado de $3 \mathrm{~mm}$ (Tabla 1). La epidermis es uniestratificada con cutícula algo engrosada y presencia de algunos estomas (Fig. 3F). En posición subepidérmica se ubica una hipodermis uniestratificada de células isodiamétricas con paredes primarias delgadas. La pared del estilo está formada por parénquima con conductos secretores y pequeños haces vasculares concéntricos anficribales (Fig. 3C), ambos son numerosos, pero estadísticamente diferentes entre las especies (Tabla 1). El centro del estilo presenta tejido transmisor y canal estilar, y muestra diferentes características según la zona del estilo (Fig. 3B, E, H).

En la zona del estilo alto o estigmático la pared estilar es angosta, de 0,71 y $0,81 \mathrm{~mm}$ en cada especie (Tabla 1, Fig. 3B). Presenta gran cantidad de conductos de tamaño notable, ubicados en un parénquima compacto por debajo de la epidermis externa, así como también en la zona cercana al tejido de transmisión. Los hacecillos concéntricos se ubicaron más cercanos a la epidermis interna (Fig. 3C); esta última, si bien no se observa definida, presenta ondulaciones bien marcadas en forma de crestas (Fig. 3B-C). El tejido transmisor es muy laxo, lo que dificultó su análisis (Fig. 3B). En esta porción del estilo se observaron diferencias significativas entre ambas especies en todas las variables indagadas, siendo mayores en $T$. candicans (Tabla 1).

En la zona del estilo medio la pared estilar es de mayor grosor que en el estilo alto, de 0,92 y 0,85 $\mathrm{mm}$ para cada especie, y por el contrario el tejido transmisor es comparativamente menor (Tabla 1, Fig. 3D-F). La pared presenta mayor cantidad de estratos celulares, el parénquima es compacto y los hacecillos se ubican aproximadamente en el centro (Fig. 3E-F). Los conductos secretores son menos numerosos (Tabla 1) y se encuentran en mayor proporción en la zona interna de la pared, entre los hacecillos y la epidermis interna (Fig. 3F); esta última presenta pequeñas ondulaciones, producidas por la presencia de los conductos (Fig. 3E-F). El tejido transmisor es más compacto y presenta una epidermis secretora bien definido, con gran cantidad de papilas recubriendo el canal estilar (Fig. 3D-E).

Los análisis estadísticos en esta porción del estilo mostraron diferencias significativas para dos variables de las cinco observadas, en el espesor de la pared estilar y en la cantidad de conductos fueron mayores en $T$. candicans. Asimismo, las variables restantes resultaron mayores para $T$. candicans (Tabla 1). 


\section{G. Fernández et al. - Morfo-anatomía de Trichocereus (Cactaceae)}
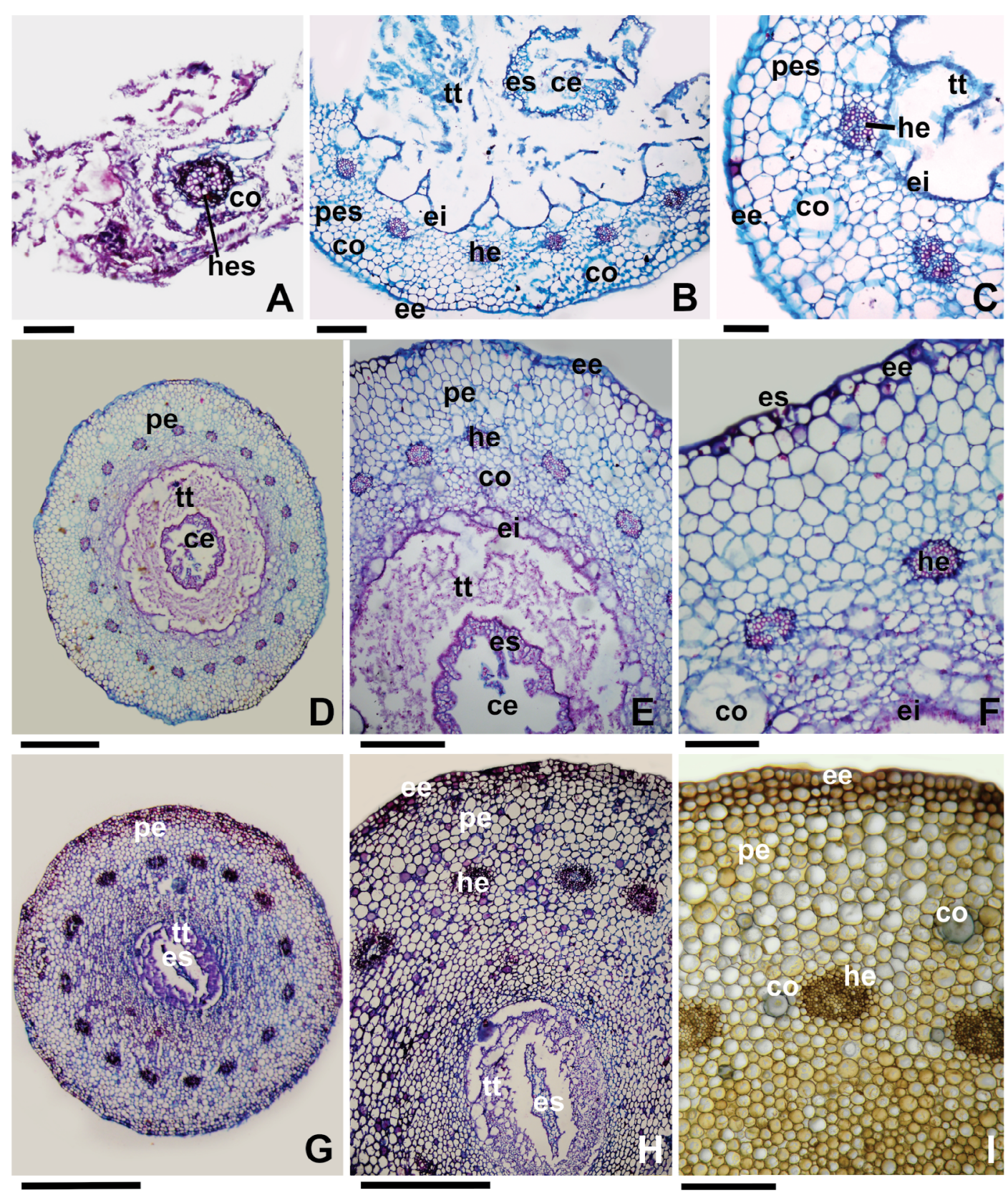

Fig. 3. Estigma y estilo de Trichocereus en corte transversal. A-F: T. candicans. G-I: T. pseudocandicans. A: Lóbulo estigmático con un haz vascular. B: Estilo alto, pared, tejido transmisor y canal estilar. C: Detalle de la pared en zona del estilo alto, se observan hacecillos estilares y conductos secretores. D: Estilo medio, vista completa. E: Detalle de la pared en zona del estilo medio, tejido transmisor y canal estilar. F: Detalle de pared estilar en zona de estilo medio. G: Estilo bajo, vista completa. H: Detalle de pared estilar en zona de estilo bajo. I: Detalle en preparado temporario de zona externa de pared del estilo, mostrando pequeños conductos. Abreviaturas= es: estoma, ce: canal estilar, co: conducto secretor, ee: epidermis externa, ei: epidermis interna, ec: epidermis del canal estilar, he: hacecillo estilar, hes: hacecillo estigmático, pe: pared estilar, tt: tejido transmisor. Escalas= D, G: 1 mm. B, E, H: $500 \mu \mathrm{m}$. C, F, I: $200 \mu \mathrm{m}$. A: $100 \mu \mathrm{m}$. 
En la zona del estilo bajo u ovárico la pared estilar es notablemente más gruesa que en las restantes zonas, de 1,11 y 1,01 $\mathrm{mm}$ para cada especie (Tabla 1, Fig. 3G-H). En contraste, el tejido de transmisión y el canal estilar son muy reducidos (Fig. $3 \mathrm{H}$ ). La pared estilar en esta porción presenta gran cantidad de estratos de células parenquimáticas, muy compactas, las que disminuyen su tamaño en la zona interna de la pared. La epidermis interna es poco diferenciada, pero sin ningún tipo de ondulación (Fig. $3 \mathrm{H}$ ). Los conductos son menos numerosos (Tabla 1) y más pequeños que en las zonas restantes (Fig. 3I).

Cuantitativamente, el estilo bajo de ambas especies resultaron similares, ya que no se encontraron diferencias significativas en ninguna variable analizada. Asimismo, todas presentaron valores mayores en $T$. candicans, a excepción del número de conductos secretores que fue mayor en T. pseudocandicans (Tabla 1).

Receptáculo: también llamado hipanto o pericarpelo, es un tejido de origen caulinar que recubre al ovario. Se encuentra formado por células parenquimáticas grandes, alargadas, dispuestas de forma perpendiculares a la epidermis, similar al parénquima en empalizada del tallo pero sin cloroplastos (Fig. 4B). Los hacecillos son escasos y reducidos, dispersos sin un patrón definido. Se observaron en esta zona numerosos conductos de gran tamaño (Fig. 4B-C), además de idioblastos con cristales tipo drusas, prismáticos y conglomerados. La epidermis e hipodermis estuvieron presentes, con características similares a las descriptas para escamas, aunque con un mayor espesor (Fig. 4 B, Tabla 1).

Los análisis estadísticos no mostraron diferencias significativas entre las especies en las mediciones tomadas (Tabla 1), aunque el grosor de la epidermis e hipodermis fue mayor en T. pseudocandicans y el ancho del receptáculo mayor en $T$. candicans.

Ovario: es multicarpelar y de placentación parietal (Fig. 4A). La pared del ovario está formada por parénquima compacto, con células notablemente más pequeñas e isodiamétricas. No se observaron conductos, ni cristales (Fig. 4C y D). El receptáculo y la pared del ovario presentan características distintivas pero se hallan íntimamente soldados, entre ellos se observó una zona de transición, con numerosos hacecillos colaterales (Fig. 4C). Cerca de la zona de las placentas, las células parenquimáticas son aún más pequeñas y con numerosos haces que inervan las placentas y funículos (Fig. 4D). Los óvulos son numerosos (Fig. 4A, D), circinótropos (Fig. $4 \mathrm{E})$, bitégmicos, crasinucelados y se encuentran agrupados en ramilletes de entre 8 a 12 óvulos, unidos por sus largos funículos (Fig. 4F).

Los análisis estadísticos no mostraron diferencias significativas entre las especies en el ancho de la pared del ovario (Tabla 1), aunque fue algo mayor en $T$. candicans.

Fruto: luego de la fecundación, la pared del fruto comienza a aumentar su espesor; el receptáculo o hipanto da lugar al clamidocarpo, mientras que la pared del ovario forma el pericarpo (Fig. $4 \mathrm{G})$, ambos tejidos son notoriamente diferentes, pero íntimamente fusionados. En general, el fruto joven presenta características anatómicas similares a las descriptas para el receptáculo y el ovario. Se destaca la epidermis con paredes celulares más engrosadas, el clamidocarpo con un parénquima algo laxo y con mayor cantidad de conductos (Fig. 4H). El pericarpo es más compacto, con haces de mayores dimensiones y sin conductos secretores (Fig. 4G, I). En las semillas el embrión se encontró en un estado de incipiente desarrollo, mientras que el episperma se destacó por sus grandes células con paredes lignificadas (Fig. 4I).

Cuantitativamente, se encontraron diferencias significativas entre las especies en el espesor de la hipodermis y del pericarpo, siendo mayores en T. peseudocandicans (Tabla 1).

\section{Análisis Cuantitativos}

De las 43 variables analizadas (Tabla 1), solo $12(27,9 \%)$ evidenciaron diferencias estadísticamente significativas entre las especies. De ellas, 8 variables mostraron mayores valores en $T$. candicans, particularmente en la parte alta y media del estilo. Por su parte, sólo 4 variables fueron mayores en $T$. pseudocandicans, en el estigma y fruto joven.

Esta misma tendencia se observó si se tienen en cuenta los resultados crudos, por lo que, en rasgos generales la flor de $T$. candicans es algo mayor que la de T. pseudocandicans (Tabla 1). 


\section{G. Fernández et al. - Morfo-anatomía de Trichocereus (Cactaceae)}
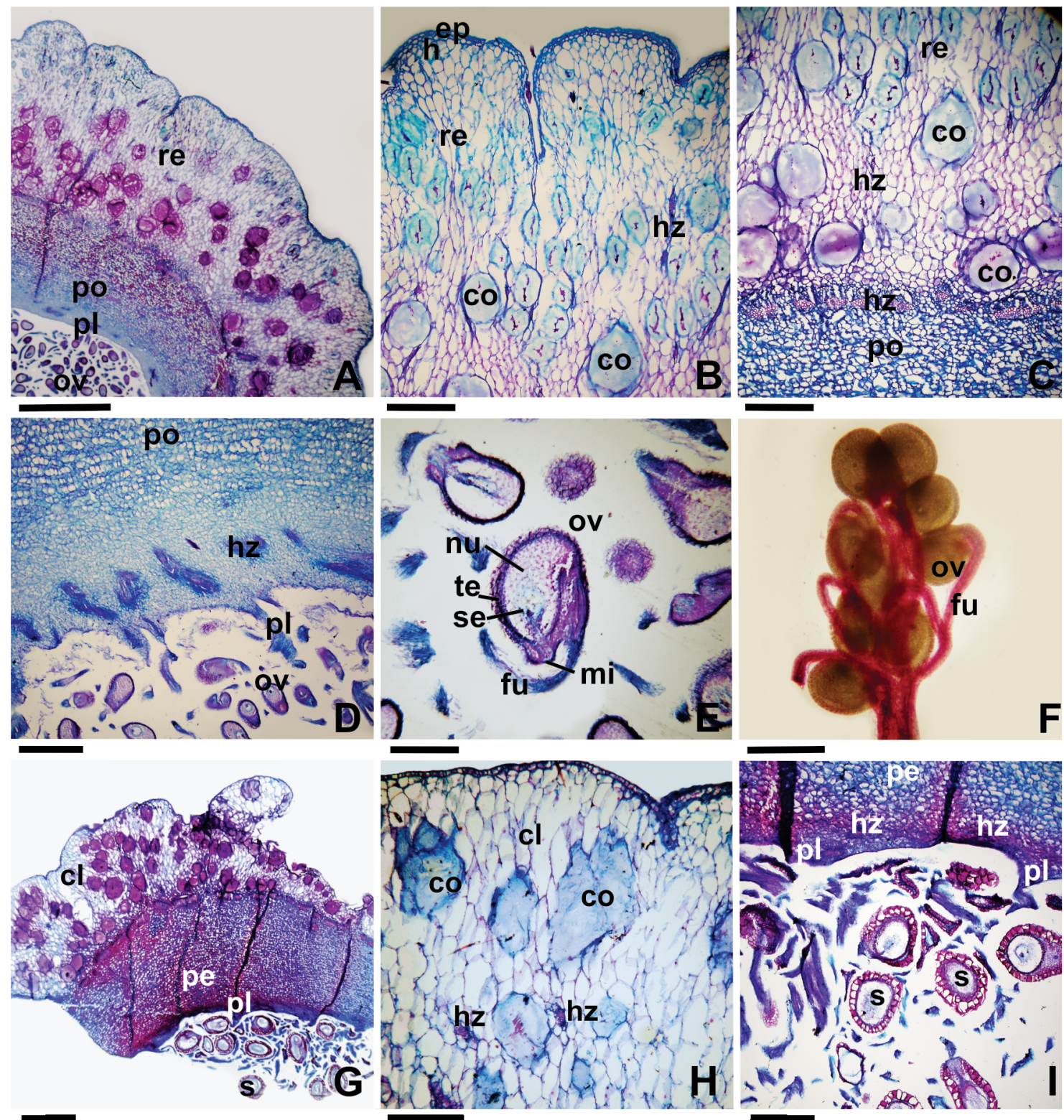

Fig. 4. Receptáculo, ovario y fruto de Trichocereus en corte transversal. A-E: T. candicans. F-I: T. pseudocandicans. A: Vista completa de la pared del ovario y receptáculo. B: Detalle del receptáculo, con gran cantidad de conductos. C: Zona de transición entre receptáculo y pared del ovario. D: Zona interna del carpelo mostrando placentas y óvulos. E: Óvulos circinótropo. F: Ramillete de óvulos en preparados temporales. G: Vista del fruto, se observa clamidocarpo y pericarpo. H: Detalle de la zona del clamidocarpo donde se observan numerosos conductos secretores. I: Zona de placentas con semillas en desarrollo. Abreviaturas $=\mathrm{cl}$ : clamidocarpo, co: conducto secretor, ep: epidermis, fu: funículo, h: hipodermis, hz: haz, nu: nucela, mi: micrópilo, ov: óvulo, pe: pericarpo, pl: placenta, po: pared del ovario, re: receptáculo, s: semilla, se: saco embrionario, te: tegumento del óvulo. Escalas= A, G: 2 mm. B-D, H-I: $500 \mu \mathrm{m}$. F: $200 \mu \mathrm{m}$. E: $100 \mu \mathrm{m}$. 


\section{Discusión}

Trichocereus candicans y $T$. pseudocandicans presentan similitudes exomorfológicas en sus flores. Se destacan por su gran tamaño y la abundante pilosidad en las areolas externas, características comunes en el género Trichocereus, pero infrecuentes en el resto de la familia (Kiesling, 1978; Albesiano \& Terrazas, 2012). La presencia de pelos en vez de espinas en las flores evitaría daños a los polinizadores que se alimentan del néctar o polen (Gibson \& Nobel, 1986; Albesiano $\&$ Terrazas, 2012).

Las especies se diferencian notablemente por el color del perigonio y por el tamaño total de las flores (Kiesling, 1978), aunque, las variables morfo anatómicas analizadas mostraron que las flores de $T$. candicans son levemente mayores que las de $T$. pseudocandicans.

El gineceo presenta grandes dimensiones, con un estilo muy desarrollado y un estigma formado por numerosos lóbulos que sobresalen del alto de las anteras; de esta manera, la hercogamia existente reduciría o impediría la autogamia (Cota, 1993; Albesiano \& Terrazas, 2012). Anatómicamente, el estilo se caracteriza por la presencia de tejido de transmisión y canal estilar. El tejido de transmisión es fundamental para el desarrollo del tubo polínico ya que es rico en pectinas. Su caracterización en las especies estudiadas fue dificultosa debido a que es un tejido laxo y mucilaginoso; a diferencia de lo encontrado en Opuntia Mill., donde las células fueron redondeadas, pequeñas y compactas (Fuentes- Pérez et al., 2009). El canal estilar se observó tapizado por una epidermis papilosa, lo que también se reportó en Opuntia, Pereskia Mill. (Boke, 1968; Fuentes-Pérez et al., 2009); con la diferencia que las especies aquí estudiadas carecen del tejido parenquimatoso con cloroplastos entre el tejido de transmisión y la epidermis papilosa, que sí se encontró en Opuntia (Fuentes-Pérez et al., 2009).

El ovario ínfero de la mayoría de las Cactáceas se encuentra rodeado por el receptáculo, el cual es un tejido de origen caulinar, también llamado pericarpelo o hipanto (Anderson, 2001, PimientaBarrios \& del Castillo, 2002; Almeida et al., 2018). En las especies analizadas se observó que estos tejidos están íntimamente fusionados, incluso luego de la fecundación y formación del fruto, a diferencia de Pereskia donde aparentan estar soldados, pero realmente lo están solo en la base del ovario (Boke, 1968; Da Rosa \& De Souza., 2003). En la zona de fusión entre el receptáculo y los carpelos se pudo observar un anillo de haces colaterales, que se mantuvo en el fruto joven; se considera que estos haces son el límite entre los tejidos, y que el receptáculo es de origen caulinar y no apendicular (Fuentes-Pérez et al., 2009; Almeida et al., 2010; Almeida et al., 2018). En las especies estudiadas, los dos tejidos fueron notoriamente diferentes, tanto en la flor como en el fruto joven, lo que hace posible su diferenciación y apoya la nomenclatura utilizada en el presente trabajo. En cambio, en la mayoría de las especies de la familia, la pared del fruto es homogénea, sin que exista una marcada diferencia anatómica que limite los tejidos de diferente origen, por lo que muchos autores consideran a la pared del fruto solo como pericarpo, formada por la epidermis externa o exocarpo, la epidermis interna o endocarpo y el mesocarpo combinado (Almeida et al., 2018). Con respecto a la nomenclatura de los frutos de Cactaceae, acordamos con las investigaciones recientes que proponen el término cactidium, que corresponde a un fruto de origen mixto, pericarpelar o receptacular y ovárico, con estructuras accesorias como lo son las areolas, que puede a su vez producir hojas, cerdas, espinas, tricomas, etc. (Anderson, 2001; Almeida et al., 2018).

Los óvulos en la familia Cactaceae se consideran del tipo anátropo, aunque se han reportado algunas variaciones (Pimienta-Barrios \& del Castillo, 2002; Fuentes-Pérez et al., 2009). En las especies analizadas aquí, se observó que el funículo continúa curvándose, envolviendo el óvulo, por lo que consideramos que son del tipo circinótropo, lo que ha sido observado en pocas especies (Strittmatter $e t$ al., 2002; Almeida et al., 2010). Las características observadas de las semillas concuerdan con lo descripto para T. candicans por Cerizo et al., (2013), que reportaron semillas pequeñas $(0.4128$ $\pm 0.0058 \mathrm{mg}$ ) con tegumento rugoso, levemente irregular y de color negro brillante, escama hilar u opérculo bien definida donde se encuentra el hilo y el orificio micropilar.

Las características de la epidermis e hipodermis han sido estudiadas en el cuerpo vegetativo de muchas especies de Cactaceae; se consideran muy variables dentro de la familia, por lo que son utilizadas para la delimitación de taxones 


\section{G. Fernández et al. - Morfo-anatomía de Trichocereus (Cactaceae)}

(Gibson \& Nobel, 1986; Anderson, 2001; LozaCornejo \& Terrazas, 2003; Terrazas-Salgado et al., 2005; Soffiatti \& Angyalossy, 2007). En conjunto, cumplen una importante función de protección, retención de agua y disminución de la radiación solar que llega al clorénquima subyacente, siendo una importante adaptación a ambientes áridos (Loza-Cornejo \& Terrazas, 2003; Vázquez-Sánchez et al., 2015). Para T. candicans se han reportado las características de la epidermis en plántulas, siendo esta no colenquimatosa, con cutícula fina, presencia de drusas y estomas paralelocítico; sin mención a la hipodemis (Cenizo el al., 2013). En los órganos reproductivos, estos tejidos también cumplirían funciones similares, ayudando a la economía hídrica de la flor y el fruto en formación, nuestras observaciones coinciden parcialmente con las encontradas en Opuntia (Fuentes-Pérez et al., 2009) en las que se reportaron en el receptáculo o pericarpelo entre dos u seis estratos de hipodermis $\mathrm{y}$ en los tépalos una epidermis colenquimatosa y colénquima subyacente.

La presencia de conductos o canales secretores mucilaginosos coincide con lo expresado para las cactáceas por Gibson \& Nobel (1986); Anderson, (2001), Soffiatti \& Angyalossy, (2007). Los mucílagos son polisacáridos similares a la pectina solubles en agua (Cárdenas et al., 1997); son altamente hidrófilos por lo que afectan las relaciones hídricas en la planta y se los relaciona con el almacenamiento de agua. Junto con la presencia de cristales, constituyen una importante adaptación a los ambientes xerofíticos (Gibson \& Nobel, 1986; Anderson, 2001, Terrazas-Salgado et al., 2005). En las flores estos compuestos también intervienen en la economía del agua, siendo además un importante impedimento contra la depredación; en los frutos maduros la presencia de gran cantidad de mucilago rodeando las semillas se considera una adaptación importante que protege al embrión de las altas temperaturas y la desecación (Almeida et al., 2018)

En la familia Cactaceae, los nectarios pueden ser de los tipos disco, embudo o surco y cámara nectarial que corresponden a los nectarios receptacular, anular e hipantial. Sin embargo, existen estadios intermedios o poco claros por lo que concordamos con Fuentes-Pérez et al. (2009) y Almeida et al., (2018) en que es necesario de un estudio comparativo del desarrollo de los nectarios con la finalidad de definir este carácter con posible valor sistemático. Las especies de Trichocereus pueden o no presentar nectarios, siendo en general del tipo cámara nectarial (Kiesling, 1978; Albesiano, 2015). A pesar de que se ha reportado con anterioridad en $T$. candicans, no así en $T$. pseudocandicans, en el presente trabajo no se pudo confirmar su presencia y consideramos que son necesarias nuevas investigaciones específicas para dilucidar este interesante aspecto. Asimismo, se pretende continuar con el estudio de los órganos reproductivos masculinos, ya que la cantidad y disposición de los estambres, así como su morfoanatomía, puede ayudar a dilucidar la identidad taxonómica de las especies aquí estudiadas.

\section{Consideraciones taxonómicas y reproductivas}

La mayoría de las especies de Trichocereus poseen flores grandes, blancas y nocturnas, que abren al atardecer, permanecen en antesis toda la noche y hasta unas horas más del día siguiente. Por el contrario, las flores coloridas y diurnas pueden permanecer abiertas por dos o tres días, son menos comunes, encontrándose en T. tarijensis (Vaupel) Werderm., T. andalgalensis (F.A.C. Weber) Hosseus, T. rowleyi (Friedrich) R. Kiesling, T. huascha (F.A.C. Weber) Hosseus, T. vatteri y T. pseudocandicans (Kiesling, 1978). Albesiano \& Terrazas (2012) presentaron un análisis filogenético donde se confirma que el carácter plesiomórfico son las flores blancas; las flores coloridas aparecen como una inversión solamente en T. tarijensis y en el clado de T. andalgalensis-T. schickendantzii, sin referencia a las restantes especies. En el árbol filogenético propuesto, $T$. candicans se encuentra alejada de estas tres especies de flores coloridas. Por lo que, si inferimos que T. pseudocandicans es una especie derivada de $T$. candicans, el carácter flor diurna debería haber aparecido de manera independiente muchas veces en el género.

Por el contrario, y teniendo en cuenta la gran similitud anatómica de las flores reportada en el presente trabajo, se podría considerar a los representantes de flores coloridas como subespecies de las de flores blancas, apoyando la teoría de Albesiano (2015), o un híbrido, según lo propuesto por Kiesling (1987). O bien, simplemente, que los diferentes caracteres florales sean variedades dentro de la población natural, planteando la posibilidad de que la diversidad sea el resultado de una gran variabilidad fenotípica, influenciada por las 
características particulares que presentan las Sierras de Famatina. Hipótesis similares fueron planteadas en algunas especies de Echinopsis sensu lato en las que se corroboró que presentan una gran diversidad floral (Schlumpberger et al., 2009).

Los diferentes síndromes florales en Trichocereus son sin duda un importante rasgo taxonómico, pero también debe ser analizado en profundidad en relación a la biología reproductiva, ya que en general las especies de este género son auto-incompatibles (De Viana et al., 2001; Ortega-Baes et al., 2010). Se ha reportado para algunas especies de flores blancas una alta diversidad de visitantes florales, tanto nocturnos como diurnos, aunque solo las polillas y abejas serían polinizadores efectivos (De Viana et al., 2001; Schlumpberger \& Badano, 2005; OrtegaBaes et al., 2010; Lemaitre et al., 2014); en tanto que, ningún estudio reproductivo se llevó a cabo con especies de flores coloridas. Podemos inferir que $T$. pseudocandicans y $T$. candicans también presentarían diversos visitantes pero es probable que no coincidan en los polinizadores efectivos, por las diferencias en color de la flor, horarios de apertura y duración de la antesis y distribución geográfica. Frente a la escasez de estudios de este tema, planteamos la necesidad de seguir con las investigaciones en este grupo de plantas, para intentar esclarecer tanto su biología reproductiva, como sus relaciones de parentesco.

\section{Conclusiones}

Las entidades se diferenciaron por el color del perigonio y el tamaño total de las flores. Anatómicamente, el perigonio, el gineceo y el fruto joven fueron similares. En los análisis cuantitativos, solo el $27,9 \%$ de las variables resultaron con diferencias estadísticamente significativas, siendo las flores de $T$. candicans levemente mayores que las de T. pseudocandicans. La gran similitud encontrada reafirma el alto grado de relación entre los taxones, siendo posible que T. pseudocandicans sea una subespecie o híbrido de $T$. candicans y otra especie de Trichocereus, siendo necesario otro tipo de estudios para conclusiones más contundentes. Por otro lado, los diferentes rasgos florales podrían ser el resultado de una gran variabilidad fenotípica, influenciada por las características particulares que presentan las Sierras de Famatina.

\section{Contribución DE los AUtores}

MLLP recolectó el material de campo, GF y AP realizaron los preparados histológicos y tomaron fotomicrografías. GF, LS y ND realizaron las observaciones micro y macroscópicas y analizaron las variables cuantitativas. Todos los autores participaron en la escritura del manuscrito.

\section{Agradecimientos}

Los autores agradecemos el financiamiento a la Secretaría de Ciencia y Tecnología, Universidad Nacional de Córdoba (SeCyT-UNC), al Fondo para la Investigación Científica y Tecnológica (FONCYT) y al Consejo Nacional de Investigaciones Científicas y Técnicas (CONICET). Además, agradecemos a los revisores por sus comentarios y sugerencias, los cuales han ayudado sustancialmente a la mejora de nuestro manuscrito.

\section{Bibliografía}

AAGESEN, L., M. BENA, S. NOMDEDEU, A. PANIZZA, R. LÓPEZ \& F. ZULOAGA. 2012. Areas of endemism in the Southern Central Andes. Darwiniana 50: 218-251. ACEÑOLAZA, P. 1996. Vegetación. In: ACEÑOLAZA, F., H. MILLER \& A. TOSELLI (eds.), Geología del sistema de Famatina. pp.19-22. Münchner Geologische Hefte 19 (Reihe A). Múnich.

ALBESIANO, S. \& T. TERRAZAS. 2012. Cladistic analysis of Trichocereus (Cactaceae: Cactoideae: Trichocereeae) based on morphological data and chloroplast DNA sequences. Haseltonia 17: 3-23. https://doi.org/10.2985/1070-0048-17.1.2.

ALBESIANO, S. 2015. Taxonomía y filogenia de Trichocereus (Berg.) Riccob. TrichocereeaeCactaceae). (Tesis doctoral). Universidad Nacional de La Plata. La Plata, Argentina.

ALMEIDA, O., A. PAOLI \& L. SOUZA. 2010. Morfoanatomía de la flor de Epiphyllum phyllanthus (Cactaceae). Rev. Mex. Biodivers. 81: 65-80.

ALMEIDA, O., L. SOUZA, A. PAOLI, A. DAVIS \& J. COTA-SÁNCHEZ. 2018. Pericarp development in fruit of epiphytic cacti: implications for fruit classification and macro-morphology in the Cactaceae. Botany 96: 621-635. https://doi.org/10.1139/cjb-2018-0074. 


\section{G. Fernández et al. - Morfo-anatomía de Trichocereus (Cactaceae)}

ANDERSON, E. 2001. The Cactus Family. Timber Press. Portland, Oregon.

BARBOZA, G., J.J. CANTERO, F. CHIARINI1, J. CHIAPELLA, S. FREIRE, C. NUÑEZ, V. PALCHETTI \& L. ARIZA ESPINAR. 2016. Vascular plants of Sierra de Famatina (La Rioja, Argentina): an analysis of its biodiversity. Phytotaxa 248: 1-123. http://dx.doi. org/10.11646/phytotaxa.248.1.1.

BOKE, N. 1968. Structure and development of the flower and fruit of Pereskia diaz-romeroana. Amer. J. Bot. 55: 1254-1260. https://doi.org/10.1002/j.1537-2197.1968.tb07494.x.

CÁRDENAS, A., I. HIGUERA-CIAPARA \& F.M. GOYCOOLEA. 1997. Rheology and aggregation of cactus (Opuntia ficus-indica) mucilage in solution. $J$. Prof. Assoc. Cactus Dev. 2: 152-159.

https://doi.org/10.31055/1851.2372.v48.n3-4.7556.

CENIZO, V., M. MAZZOLA, B. CORRÓ MOLAS \& A. KIN. 2013. Características morfológicas y anatómicas de las plántulas de Trichocereus candicans (Cactaceae). Bol. Soc. Argent. Bot. 48: 443-451. https://doi.org/10.31055/1851.2372.v48.n3-4.7546

COTA, J. 1993. Pollination syndromes in the genus Echinocereus: A review. Cact. Succ. Journ. 65: 19- 26.

DAROSA, S. \& L. SOUZA. 2003. Morfo-anatomia do fruto (hipanto, pericarpo e semente) em desenvolvimento de Pereskia aculeata Miller (Cactaceae). Acta Sci. Biol. 25: 415-428.

https://doi.org/ 10.4025/actascibiolsci.v25i2.2046.

DE VIANA M., P. ORTEGA-BAES, M. SARAVIA, E. BADANO \& B. SCHLUMP- BERGER. 2001. Biologia floral y polinizadores de Trichocereus pasacana (Cactaceae) en el Parque Nacional Los Cardones, Argentina. Rev. Biol. Trop. 49: 279-285.

DI RIENZO, J., F. CASANOVES, M. BALZARINI, L. GONZÁLEZ, M. TABLADA \& C. ROBLEDO. 2017. Infostat, versión 2017, Grupo Infostat, F. C. A. (UNC), Córdoba, Argentina.

FUENTES-PÉREZ, M., T. TERRAZA \& S. ARIAS. 2009. Anatomía floral de cinco especies de Opuntia (Opuntioideae, Cactaceae) de México. Polibotánica 27: 89-102.

GIBSON, A. \& P. NOBEL (ed.). 1986. The cactus primer. Harvard University Press. Cambridge.

GODOY-BÜRKI, A., P. ORTEGA-BAES, J. SAJAMA \& L. AAGESEN. 2014. Conservation priorities in the Southern Central Andes: mismatch between endemism and diversity hotspots in the regional flora. Biodivers. Conserv. 23: 81-107. http://dx.doi.org/10.1007/s10531-013-0586-1.
GRIFFITH, M. \& J. PORTER. 2009. Phylogeny of Opuntioideae (Cactaceae). Int. J. Plant Sci. 170: 107-116. https://doi.org/10.1086/593048.

HUNT, D., N. TAYLOR \& G. CHARLES (eds.). 2006. The New Cactus Lexicon. DH Books, Milborne Port.

KIESLING, R. 1978. El género Trichocereus (Cactaceae). Apéndice I: Las especies de la República Argentina. Darwiniana 21: 263-330.

KIESLING, R, J. LAROCCA, L. FAÚNDEZ, D. METZING \& S. ALBESIANO. 2008. Cactaceae. In: ZUOLAGA F., O. MORRONE \& M. BELGRANO (eds.) Catálogo de las Plantas Vasculares del Cono Sur. Vol. 2, pp. 1715-1830. Missouri Botanical Garden Press, Saint Louis.

KRAUS, J., H. DE SOUSA, M. REZENDE, N. CASTRO, C. VECCHI \& R. LUQUE. 1998. Astra Blue and Basic Fuchsin double staining of plant materials. Biotech. Histochem. 73: 235-243.

LEMAITRE A., C. PINTO \& H. NIEMEYER. 2014. Generalized pollination system: are floral traits adapted to different pollinators? Arthropod-Plant Interact. 8: 261-272. https://doi.org/10.1007/s11829-014-9308-1.

LOZA-CORNEJO, S. \& T. TERRAZAS. 2003. Epidermal and hypodermal characteristics in North American Cactoideae (Cactaceae). J. Plant Res. 116: 27-35. https://doi.org/10.1007/s10265-002-0066-2

NYFFELER, R. 2002. Phylogenetic relationships in the Cactus family (Cactaceae) based on evidence from trnK/matK and trnLtrnF sequences. Am. J. Bot. 89: 312-326. https://doi.org/10.3732/ajb.89.2.312.

ORTEGA-BAES P., M. SARAVIA, S. SÜHRING, H. GODÍNEZ ALVAREZ \& M. ZAMAR. 2010. Reproductive biology of Echinopsis terscheckii (Cactaceae): the role of nocturnal and diurnal pollinators. Plant Biol. 13: 33-40. https://doi.org/10.1111/j.1438-8677.2010.00332.x

PIMIENTA-BARRIOS, E. \& R. DEL CASTILLO. 2002. Reproductive Biology. In: NOBEL, P (ed.). Cacti, biology and uses, pp 163-183. University of California Press, Los Angeles.

SCHLUMPBERGER B. \& E. BADANO. 2005. Diversity of floral visitors to Echinopsis atacamensis subsp. pasacana (Cactaceae). Haseltonia 11: 18-26.

SCHLUMPBERGER B., A. COCUCCI, M. MORE, A. SERSIC \& R. RAGUSO. 2009. Extreme variation in floral characters and its consequences for pollinator attraction among populations of an Andean cactus. Ann. Bot. 103: 1489-1500. https://doi.org/10.1093/aob/mcp075. 
SCHLUMPBERGER, B. \& S. RENNER. 2012. Molecular phylogenetics of Echinopsis (Cactaceae): Polyphyly at all levels and convergent evolution of pollination modes and growth forms. Amer. J. Bot. 99: 1335-1349. https://doi.org/10.3732/ajb.1100288.

SOFFIATTI, P. \& V. ANGYALOSSY. 2007. Anatomy of Brazilian Cereeae (subfamily Cactoideae, Cactaceae): Arrojadoa Britton \& Rose, Stephanocereus A. Berger and Brasilicereus Backeberg. Acta Bot. Bras. 21: 813-822. https://doi.org/10.1590/S0102-33062007000400006.

STRITTMATTER, L., V. NEGRÓN-ORTIZ \& R. HICKEY. 2002. Subdioecy in Consolea spinosissima (Cactaceae): breeding system and embryological studies. Am. J. Bot. 89: 1373-1387. https://doi.org/10.3732/ajb.89.9.1373
TERRAZAS-SALGADO, T., S. LOZA-CORNEJO \& H. ARREOLA-NAVA. 2005. Stem anatomy of the species of the genus Stenocereus (Cactaceae). Acta Bot. Venez. 28: 321-336.

VÁZQUEZ-SÁNCHEZ, M., T. TERRAZAS \& S. ARIAS. 2015. Comparative morphology and anatomy of Backebergia militaris (EchinocereeaeCactaceae) cephalium. Plant. Syst. Evol. 302: 245256. https://doi.org/10.1007/s00606-015-1256-4.

YOUNG, K., C., ULLOA ULLOA, J. LUTEYN \& S. KNAP. 2002. Plant evolution and endemism in Andean South America: an introduction. Bot. Rev. 68: 4-21. http://dx.doi.org/10.1663/00068101(2002)068[0424:E]2.0.CO;2.

ZARLAVSKY, G. (ed). 2014. Histología Vegetal: técnicas simples y complejas, 1era ed. Sociedad Argentina de Botánica. Gráfica Arte, Buenos Aires. 